\title{
On the local theta correspondence and $R$-groups
}

\author{
Atsushi Ichino
}

\begin{abstract}
For the reductive dual pair $(U(n, n), U(n, n))$ over a $p$-adic field, we study the local theta correspondence for certain tempered representations in terms of $R$-groups. In the case we consider, the Langlands parameter is preserved, but, a twist occurs in the $L$-packet. Moreover this twist is determined by root numbers.
\end{abstract}

\section{Introduction}

Let $G=U(n, n)$ be the quasi-split unitary group in $2 n$ variables over a $p$-adic field and $\pi$ an irreducible admissible representation of $G$. Then the local theta correspondence asserts that an irreducible admissible representation $\theta(\pi)$ of $G^{\prime}=U(n, n)$ which satisfies

$$
\operatorname{Hom}_{G \times G^{\prime}}(\omega, \tilde{\pi} \otimes \theta(\pi)) \neq 0
$$

is uniquely determined if it exists. Here $\omega$ is the Weil representation of $G \times G^{\prime}$ and $\tilde{\pi}$ is the contragredient representation of $\pi$. This correspondence was conjectured by Howe [How79] and proved by Waldspurger [Wal90] when $p \neq 2$. However, it still remains difficult to describe the relation between $\pi$ and $\theta(\pi)$ explicitly. In this paper, we will determine the correspondence for certain tempered representations. It turns out that root numbers play an important role as in the epsilon dichotomy by Harris, Kudla and Sweet [HKS96].

More precisely, let $F$ be a $p$-adic field with $p \neq 2$ and $E$ a quadratic extension of $F$. Fix $\delta \in E^{\times}$ such that $\operatorname{tr}_{E / F}(\delta)=0$. We realize $G$ (respectively $G^{\prime}$ ) as the isometry group of the hermitian (respectively skew-hermitian) form given by

$$
\left(\begin{array}{cc}
\mathbf{0}_{n} & -\delta \mathbf{1}_{n} \\
\delta \mathbf{1}_{n} & \mathbf{0}_{n}
\end{array}\right) \quad\left(\text { respectively }\left(\begin{array}{cc}
\mathbf{0}_{n} & \mathbf{1}_{n} \\
-\mathbf{1}_{n} & \mathbf{0}_{n}
\end{array}\right)\right)
$$

In fact, $G=G^{\prime}$. Then we have the Weil representation $\omega$ of $G \times G^{\prime}$ for a fixed non-trivial additive character $\psi_{F}$ of $F$. Note that $\omega$ also depends on the choice of $\delta$.

Let $\pi$ be an irreducible tempered representation of $G$. Then $\pi$ is realized as an irreducible component of an induced representation $I(\sigma)=\operatorname{Ind}_{P}^{G}(\sigma)$, where $P$ is a parabolic subgroup of $G$ and $\sigma$ is a discrete series representation of the Levi component $L$ of $P$. In this paper, we assume that $L \simeq G L_{n_{1}}(E) \times \cdots \times G L_{n_{t}}(E)$ with $n=n_{1}+\cdots+n_{t}$. By the induction principle, which is due to Kudla [Kud86] and extended to the general cases in [MVW87, Chapter 3], we see that $\theta(\pi)$ is an irreducible component of the induced representation $I^{\prime}(\sigma)=\operatorname{Ind}_{P^{\prime}}^{G^{\prime}}(\sigma)$ if $\sigma$ is supercuspidal. Here we regard $P^{\prime}=P$ as a parabolic subgroup of $G^{\prime}$. However, this principle does not determine which component corresponds to $\pi$.

On the other hand, the irreducible components of $I(\sigma)$ are parameterized by the theory of $R$-groups, which is due to Harish-Chandra [Sil79b], Knapp and Stein [KS80], and Silberger

Received 18 September 2002, accepted in final form 13 March 2003.

2000 Mathematics Subject Classification 11F27, $22 \mathrm{E} 50$.

Keywords: local theta correspondence, $R$-groups, root numbers.

Partially supported by JSPS Research Fellowships for Young Scientists.

This journal is (C) Foundation Compositio Mathematica 2004. 


\section{A. ICHINO}

[Sil78, Sil79a]. We now recall the computation of $R$-groups by Goldberg [Gol95]. Write $\sigma=\sigma_{1} \otimes$ $\cdots \otimes \sigma_{t}$ with a discrete series representation $\sigma_{i}$ of $G L_{n_{i}}(E)$ for $1 \leqslant i \leqslant t$. Let $W$ be the Weyl group of $G$ with respect to the split component of the center of $L$. Note that $W$ is isomorphic to a subgroup of $(\mathbb{Z} / 2 \mathbb{Z})^{t} \rtimes \mathfrak{S}_{t}$. Let $R$ be the subgroup of $W$ generated by the sign change $r_{i}$ at the $i$ th component for all $i \in \mathfrak{I}$, where $\mathfrak{I} \subset\{1, \ldots, t\}$ consists of $i$ such that:

- $\sigma_{i} \simeq{ }^{t} \bar{\sigma}_{i}^{-1}$;

- $\sigma_{i} \not \sigma_{j}$ for all $j>i$;

- the Asai $L$-function $L_{\text {Asai }}\left(s, \sigma_{i}\right)$ is holomorphic at $s=0$.

Note that $R \simeq(\mathbb{Z} / 2 \mathbb{Z})^{\sharp \mathcal{I}}$ is abelian. Then there exists an algebra isomorphism

$$
\begin{aligned}
\mathbb{C}[R] & \longrightarrow \operatorname{End}_{G}(I(\sigma)), \\
r & \longmapsto \mathcal{N}(r, \sigma),
\end{aligned}
$$

where $\mathcal{N}(r, \sigma)$ is obtained from the normalized intertwining operator. In particular, $I(\sigma)$ has the irreducible decomposition in the form

$$
I(\sigma)=\bigoplus_{\kappa \in \hat{R}} \pi_{\kappa}
$$

where $\hat{R}$ is the character group of $R$ and

$$
\pi_{\kappa}=\{f \in I(\sigma) \mid \mathcal{N}(r, \sigma) f=\kappa(r) f \text { for all } r \in R\} .
$$

Moreover we may assume that $\pi_{1}$ is $\chi$-generic. Here $\chi$ is a fixed non-degenerate character of the unipotent radical of the standard Borel subgroup of $G$. Similarly, $I^{\prime}(\sigma)$ has the irreducible decomposition in the form

$$
I^{\prime}(\sigma)=\bigoplus_{\kappa^{\prime} \in \hat{R}^{\prime}} \pi_{\kappa^{\prime}}^{\prime}
$$

where

$$
\pi_{\kappa^{\prime}}^{\prime}=\left\{f^{\prime} \in I^{\prime}(\sigma) \mid \mathcal{N}\left(r^{\prime}, \sigma\right) f^{\prime}=\kappa^{\prime}\left(r^{\prime}\right) f^{\prime} \text { for all } r^{\prime} \in R^{\prime}\right\} .
$$

Here we regard $W^{\prime}=W$ as the Weyl group of $G^{\prime}, r_{i}^{\prime}=r_{i}$ as an element of $W^{\prime}, R^{\prime}=R$ as a subgroup of $W^{\prime}$, and $\mathcal{N}\left(r^{\prime}, \sigma\right)$ as an element of $\operatorname{End}_{G^{\prime}}\left(I^{\prime}(\sigma)\right)$. We remark that $\pi_{1}^{\prime}$ is also $\chi$-generic if we identify $\chi$ with a non-degenerate character of the unipotent radical of the standard Borel subgroup of $G^{\prime}$.

Then our main result is as follows (cf. Theorem 4.1).

Theorem. Let $\kappa \in \hat{R}$. Then $\theta\left(\pi_{\kappa}\right)=\pi_{\kappa^{\prime}}^{\prime}$ where

$$
\kappa^{\prime}\left(r_{i}^{\prime}\right)=\kappa\left(r_{i}\right) \cdot \epsilon\left(1 / 2, \sigma_{i}, \psi_{F} \circ \operatorname{tr}_{E / F}\right) \omega_{\sigma_{i}}(\delta)^{-1}
$$

for $i \in \mathfrak{I}$. Here $\omega_{\sigma_{i}}$ denotes the central character of $\sigma_{i}$.

In the proof of the main theorem, we construct explicitly an element $T$ of

$$
\operatorname{Hom}_{G \times G^{\prime}}\left(\omega \otimes I(\sigma), I^{\prime}(\sigma)\right)
$$

which satisfies the following conditions:

i) For each non-zero $f \in I(\sigma)$, there exists $\Phi \in \mathcal{S}$ such that

$$
T(\Phi, f) \neq 0 \text {. }
$$

ii) Let $i \in \mathfrak{I}, \Phi \in \mathcal{S}$, and $f \in I(\sigma)$, then

$$
\mathcal{N}\left(r_{i}^{\prime}, \sigma\right) T(\Phi, f)=\omega_{\sigma_{i}}(\delta)^{-1} \epsilon\left(1 / 2, \sigma_{i}, \psi_{F} \circ \operatorname{tr}_{E / F}\right) T\left(\Phi, \mathcal{N}\left(r_{i}, \sigma\right) f\right) .
$$




\section{LOCAL THETA CORRESPONDENCE AND $R$-GROUPS}

Here $\mathcal{S}$ is the space of $\omega$. We remark that condition ii is crucial for us. From these properties, one easily deduces the main theorem.

Notation. Let $F$ be a $p$-adic field with $p \neq 2$ and $E$ a quadratic extension of $F$. Throughout this paper, we fix a non-trivial additive character $\psi_{F}$ of $F$ and an element $\delta$ of $E^{\times}$such that $\operatorname{tr}_{E / F}(\delta)=0$. Let $\mathfrak{o}_{E}$ denote the maximal compact subring of $E,||$ the absolute value on $E$, and $x \mapsto \bar{x}$ the non-trivial Galois automorphism of $E$ over $F$. Define a non-trivial additive character $\psi$ of $E$ by $\psi=\psi_{F} \circ \operatorname{tr}_{E / F}$. We take the self-dual Haar measure on $E$ with respect to $\psi$.

Let $n \in \mathbb{N}$ and $\mathbf{n}=\left(n_{1}, \ldots, n_{t}\right) \in \mathbb{N}^{t}$ such that $n=n_{1}+\cdots+n_{t}$. Put

$$
\begin{aligned}
X_{n} & =\left\{x \in M_{n}(E) \mid{ }^{t} \bar{x}=x\right\}, \\
Z_{\mathbf{n}} & =\left\{z=\left(z_{i j}\right) \in M_{n}(E) \mid z_{i j}=0 \text { if } i>j\right\},
\end{aligned}
$$

where $z_{i j} \in M_{n_{i}, n_{j}}(E)$ for $1 \leqslant i, j \leqslant t$. We define a parabolic subgroup $P_{\mathbf{n}}=L_{\mathbf{n}} U_{\mathbf{n}}$ of $G L_{n}(E)$ by

$$
\begin{aligned}
& L_{\mathbf{n}}=\left\{a=\operatorname{diag}\left(a_{1}, \ldots, a_{t}\right) \mid a_{i} \in G L_{n_{i}}(E) \text { for } 1 \leqslant i \leqslant t\right\}, \\
& U_{\mathbf{n}}=\left\{u=\left(u_{i j}\right) \in Z_{\mathbf{n}} \mid u_{i i}=\mathbf{1}_{n_{i}} \text { for } 1 \leqslant i \leqslant t\right\} .
\end{aligned}
$$

For $a=\operatorname{diag}\left(a_{1}, \ldots, a_{t}\right) \in L_{\mathbf{n}}$ and $\lambda=\left(\lambda_{1}, \ldots, \lambda_{t}\right) \in \mathbb{C}^{t}$, we write

$$
|a|^{\lambda}=\left|\operatorname{det} a_{1}\right|^{\lambda_{1}} \cdots\left|\operatorname{det} a_{t}\right|^{\lambda_{t}} .
$$

Define $\rho_{\mathbf{n}} \in \mathbb{C}^{t}$ by

$$
2 \rho_{\mathbf{n}}=\left(n-n_{1}, \ldots,-n_{1}-\cdots-n_{i-1}+n_{i+1}+\cdots+n_{t}, \ldots,-n+n_{t}\right) .
$$

Then ||$^{2 \rho_{\mathbf{n}}}$ is the modulus character of $P_{\mathbf{n}}$.

\section{1. $L$ and $\epsilon$-factors for $G L_{n}$}

In this section, we review the theory of $L$ and $\epsilon$-factors for $G L_{n}$ by Godement and Jacquet [GJ72]. Let $\sigma$ be a discrete series representation of $G L_{n}(E)$. Then the standard $L$-factor $L(s, \sigma)$ is holomorphic for $\operatorname{Re}(s)>0$. For $\varphi \in \mathcal{S}\left(M_{n}(E)\right), s \in \mathbb{C}$, and a matrix coefficient $\phi$ of $\sigma$, put

$$
Z(\varphi, s, \phi)=\int_{G L_{n}(E)} \varphi(a) \phi(a)|a|^{s} d a .
$$

This integral is absolutely convergent for $\operatorname{Re}(s)>(n-1) / 2$, and has a meromorphic continuation to the whole $s$-plane. Moreover

$$
\frac{Z(\varphi, s+(n-1) / 2, \phi)}{L(s, \sigma)}
$$

is entire.

Let $\tilde{\sigma}$ be the contragredient representation of $\sigma$. Then $\check{\phi}(a)=\phi\left(a^{-1}\right)$ is a matrix coefficient of $\tilde{\sigma}$. We define the Fourier transform $\hat{\varphi} \in \mathcal{S}\left(M_{n}(E)\right)$ of $\varphi$ by

$$
\hat{\varphi}(x)=\int_{M_{n}(E)} \varphi(y) \psi(\operatorname{tr}(x y)) d y .
$$

Then the following functional equation holds:

$$
\frac{Z(\hat{\varphi}, 1-s+(n-1) / 2, \check{\phi})}{L(1-s, \tilde{\sigma})}=\epsilon(s, \sigma, \psi) \frac{Z(\varphi, s+(n-1) / 2, \phi)}{L(s, \sigma)} .
$$

Here $\epsilon(s, \sigma, \psi)$ denotes the standard $\epsilon$-factor.

We write $\bar{\sigma}(a)=\sigma(\bar{a})$ and ${ }^{t} \sigma^{-1}(a)=\sigma\left({ }^{t} a^{-1}\right)$ for $a \in G L_{n}(E)$. 


\section{A. ICHINO}

Lemma 1.1. Assume that $\sigma \simeq{ }^{t} \bar{\sigma}^{-1}$. Then

$$
\epsilon(1 / 2, \sigma, \psi) \omega_{\sigma}(\delta)^{-1}= \pm 1,
$$

where $\omega_{\sigma}$ is the central character of $\sigma$.

Proof. Since $\tilde{\sigma} \simeq{ }^{t} \sigma^{-1} \simeq \bar{\sigma}$ and $\psi=\psi_{F} \circ \operatorname{tr}_{E / F}$, we see that

$$
\epsilon(s, \tilde{\sigma}, \psi)=\epsilon(s, \bar{\sigma}, \psi)=\epsilon(s, \sigma, \psi) .
$$

On the other hand, we have

$$
\epsilon(s, \sigma, \psi) \epsilon(1-s, \tilde{\sigma}, \psi)=\omega_{\sigma}(-1)
$$

hence

$$
\epsilon(1 / 2, \sigma, \psi)^{2}=\omega_{\sigma}(-1)=\omega_{\sigma}(\delta)^{2}
$$

\section{Weil representations}

For $n \in \mathbb{N}$, let $V=V_{n}$ be the space of column vectors $E^{2 n}$ equipped with a hermitian form $($, defined by

$$
\left(x, x^{\prime}\right)={ }^{t} \bar{x}\left(\begin{array}{cc}
\mathbf{0}_{n} & -\delta \mathbf{1}_{n} \\
\delta \mathbf{1}_{n} & \mathbf{0}_{n}
\end{array}\right) x^{\prime} \in E
$$

for $x, x^{\prime} \in V$. For each $l \in \mathbb{N}$, we identify $V^{l}$ with $M_{2 n, l}(E)$, and put

$$
\left(x, x^{\prime}\right)={ }^{t} \bar{x}\left(\begin{array}{cc}
\mathbf{0}_{n} & -\delta \mathbf{1}_{n} \\
\delta \mathbf{1}_{n} & \mathbf{0}_{n}
\end{array}\right) x^{\prime} \in M_{l}(E)
$$

for $x, x^{\prime} \in V^{l}$. Let $G=G_{n}$ denote the isometry group of $(V,($, $))$, i.e.,

$$
G_{n}=\left\{g \in G L_{2 n}(E) \mid t \bar{g}\left(\begin{array}{cc}
\mathbf{0}_{n} & -\delta \mathbf{1}_{n} \\
\delta \mathbf{1}_{n} & \mathbf{0}_{n}
\end{array}\right) g=\left(\begin{array}{cc}
\mathbf{0}_{n} & -\delta \mathbf{1}_{n} \\
\delta \mathbf{1}_{n} & \mathbf{0}_{n}
\end{array}\right)\right\} .
$$

Similarly, we define $G^{\prime}=G_{n}^{\prime}$ by

$$
G_{n}^{\prime}=\left\{g^{\prime} \in G L_{2 n}(E) \mid g^{\prime}\left(\begin{array}{cc}
\mathbf{0}_{n} & \mathbf{1}_{n} \\
-\mathbf{1}_{n} & \mathbf{0}_{n}
\end{array}\right){ }^{t} \bar{g}^{\prime}=\left(\begin{array}{cc}
\mathbf{0}_{n} & \mathbf{1}_{n} \\
-\mathbf{1}_{n} & \mathbf{0}_{n}
\end{array}\right)\right\} .
$$

In fact, $G=G^{\prime}$.

For the reductive dual pair $\left(G, G^{\prime}\right)$ in $S p_{8 n^{2}}(F)$, we have the Weil representation $\omega$ of $G \times G^{\prime}$ on $\mathcal{S}=\mathcal{S}\left(V^{n}\right)=\mathcal{S}\left(M_{2 n, n}(E)\right)$ as in $[\operatorname{Kud} 94, \S 5]$. Here we take $\eta=\psi_{F}$ and $\xi=1$ with the notation in Theorem 3.1 of [Kud94]. Let $\Phi \in \mathcal{S}$ and $x \in V^{n}$. Then

$$
\omega(g, 1) \Phi(x)=\Phi\left(g^{-1} x\right)
$$

for $g \in G$. The action of $G^{\prime}$ is given by the following formulas. For $a \in G L_{n}(E)$ and $b \in X_{n}$,

$$
\begin{aligned}
\omega\left(1,\left(\begin{array}{cc}
a & \mathbf{0}_{n} \\
\mathbf{0}_{n} & { }^{t} \bar{a}^{-1}
\end{array}\right)\right) \Phi(x) & =|a|^{n} \Phi(x a), \\
\omega\left(1,\left(\begin{array}{cc}
\mathbf{1}_{n} & b \\
\mathbf{0}_{n} & \mathbf{1}_{n}
\end{array}\right)\right) \Phi(x) & =\psi(\operatorname{tr}((x, x) b) / 2) \Phi(x) .
\end{aligned}
$$

Let $1 \leqslant l \leqslant n$. Then

$$
\omega\left(1,\left(\begin{array}{cc|cc}
0 & 0 & \mathbf{1}_{l} & 0 \\
0 & \mathbf{1}_{n-l} & 0 & 0 \\
\hline-\mathbf{1}_{l} & 0 & 0 & 0 \\
0 & 0 & 0 & \mathbf{1}_{n-l}
\end{array}\right)\right) \Phi\left(x^{\prime}, x^{\prime \prime}\right)=\int_{V^{l}} \Phi\left(y, x^{\prime \prime}\right) \psi\left(\operatorname{tr}\left(y, x^{\prime}\right)\right) d y
$$




\section{LOCAL THETA CORRESPONDENCE AND $R$-GROUPS}

for $x^{\prime} \in V^{l}$ and $x^{\prime \prime} \in V^{n-l}$. Here $d y$ is the self-dual Haar measure on $V^{l}$ with respect to the pairing $\psi(\operatorname{tr}()$,$) . More precisely,$

$$
d y=|\delta|^{l n} \prod_{i, j} d y_{i j}
$$

for $y=\left(y_{i j}\right) \in V^{l}=M_{2 n, l}(E)$, where $d y_{i j}$ is the self-dual Haar measure on $E$ with respect to $\psi$.

\section{3. $R$-groups}

Let $\mathbf{n}=\left(n_{1}, \ldots, n_{t}\right) \in \mathbb{N}^{t}$ be a partition of $n$. We define a parabolic subgroup $P=L U$ of $G$ by

$$
\begin{aligned}
& L=\left\{\left(\begin{array}{cc}
a & \mathbf{0}_{n} \\
\mathbf{0}_{n} & { }^{t} \bar{a}^{-1}
\end{array}\right) \in G \mid a \in L_{\mathbf{n}}\right\}, \\
& U=\left\{\left(\begin{array}{cc}
u & * \\
\mathbf{0}_{n} & { }^{t} \bar{u}^{-1}
\end{array}\right) \in G \mid u \in U_{\mathbf{n}}\right\} .
\end{aligned}
$$

Put $\rho=\rho_{\mathbf{n}}+n / 2$. Then ||$^{2 \rho}$ is the modulus character of $P$. Let $K=G \cap G L_{2 n}\left(\mathfrak{o}_{E}\right)$ be a maximal compact subgroup of $G$. Then the Iwasawa decomposition $G=P K$ holds.

For $1 \leqslant i \leqslant t$, let $\sigma_{i}$ be a discrete series representation of $G L_{n_{i}}(E)$ on $\mathcal{V}_{i}$ with the central character $\omega_{\sigma_{i}}$. Then $\sigma=\sigma_{1} \otimes \cdots \otimes \sigma_{t}$ is a discrete series representation of $L$ on $\mathcal{V}=\mathcal{V}_{1} \otimes \cdots \otimes \mathcal{V}_{t}$. For $\lambda \in \mathbb{C}^{t}$, we write $I(\sigma, \lambda)=\operatorname{Ind}_{P}^{G}\left(\sigma||^{\lambda}\right)$. Let $W$ be the Weyl group with respect to the split component of the center of $L$, and $w \in G$ a representative for an element of $W$. For a holomorphic section $f^{(\lambda)}$ of $I(\sigma, \lambda)$, put

$$
M(w, \sigma, \lambda) f^{(\lambda)}(g)=\int_{\left(U \cap w U w^{-1}\right) \backslash U} f^{(\lambda)}\left(w^{-1} u g\right) d u .
$$

This integral is absolutely convergent if $\operatorname{Re}\left(\lambda_{1}\right) \gg \cdots \gg \operatorname{Re}\left(\lambda_{t}\right) \gg 0$, and has a meromorphic continuation to $\mathbb{C}^{t}$. By Theorem 2.1 of [Art89a], there exist meromorphic functions $r(w, \sigma, \lambda)$ of $\lambda$ such that the normalized intertwining operator

$$
N(w, \sigma, \lambda)=r(w, \sigma, \lambda)^{-1} M(w, \sigma, \lambda)
$$

is holomorphic on $\sqrt{-1} \mathbb{R}^{t}$ and that the cocycle condition

$$
N\left(w w^{\prime}, \sigma, 0\right)=N\left(w, w^{\prime} \sigma, 0\right) N\left(w^{\prime}, \sigma, 0\right)
$$

holds for representatives $w, w^{\prime} \in G$ for elements of $W$. See also [Sha90, $\S 7$ ].

We now recall the computation of $R$-groups by Goldberg [Gol95]. Let $\mathfrak{I}$ be the set of $i \in\{1, \ldots, t\}$ such that:

- $\sigma_{i} \simeq{ }^{t} \bar{\sigma}_{i}^{-1}$;

- $\sigma_{i} \not 千 \sigma_{j}$ for all $j>i$;

- $L_{\text {Asai }}\left(s, \sigma_{i}\right)$ is holomorphic at $s=0$.

Here $L_{\text {Asai }}\left(s, \sigma_{i}\right)$ is the Asai $L$-function for $\sigma_{i}$ defined by the Langlands-Shahidi method [Sha90, Gol94]. For $1 \leqslant i \leqslant t$, put $l_{i}=n_{1}+\cdots+n_{i-1}, m_{i}=n_{i+1}+\cdots+n_{t}$, and

$$
w_{i}=\left(\begin{array}{ccc|ccc}
\mathbf{1}_{l_{i}} & 0 & 0 & 0 & 0 & 0 \\
0 & 0 & 0 & 0 & \mathbf{1}_{n_{i}} & 0 \\
0 & 0 & \mathbf{1}_{m_{i}} & 0 & 0 & 0 \\
\hline 0 & 0 & 0 & \mathbf{1}_{l_{i}} & 0 & 0 \\
0 & -\mathbf{1}_{n_{i}} & 0 & 0 & 0 & 0 \\
0 & 0 & 0 & 0 & 0 & \mathbf{1}_{m_{i}}
\end{array}\right) \in G .
$$




\section{A. ICHINO}

Let $r_{i}$ be the image of $w_{i}$ in $W$ and $R$ the subgroup of $W$ generated by $r_{i}$ for all $i \in \mathfrak{I}$. For each $i \in \mathfrak{I}$, we fix an isomorphism $A_{i}: \mathcal{V}_{i} \stackrel{\sim}{\rightarrow} \mathcal{V}_{i}$ such that $A_{i}^{2}=$ id and $A_{i} \sigma_{i}(a)={ }^{t} \bar{\sigma}_{i}^{-1}(a) A_{i}$ for all $a \in G L_{n_{i}}(E)$. Then

$$
\mathcal{N}\left(r_{i}, \sigma\right)=\omega_{\sigma_{i}}(\delta) A_{i} N\left(w_{i}, \sigma, 0\right)
$$

is a self-intertwining operator of $I(\sigma)=I(\sigma, 0)$ and satisfies $\mathcal{N}\left(r_{i}, \sigma\right)^{2}=$ id. For $r=r_{i_{1}} \cdots r_{i_{k}} \in R$ with $\left\{i_{1}, \ldots, i_{k}\right\} \subset \mathfrak{I}$, put

$$
\mathcal{N}(r, \sigma)=\mathcal{N}\left(r_{i_{1}}, \sigma\right) \cdots \mathcal{N}\left(r_{i_{k}}, \sigma\right)
$$

By the theory of $R$-groups, the algebra homomorphism defined by

$$
\begin{aligned}
\mathbb{C}[R] & \longrightarrow \operatorname{End}_{G}(I(\sigma)) \\
r & \longmapsto \mathcal{N}(r, \sigma)
\end{aligned}
$$

is in fact an isomorphism. Let $\hat{R}$ denote the character group of $R$. For each $\kappa \in \hat{R}$, let

$$
\pi_{\kappa}=\{f \in I(\sigma) \mid \mathcal{N}(r, \sigma) f=\kappa(r) f \text { for all } r \in R\} .
$$

Then we see that $\pi_{\kappa}$ is irreducible and

$$
I(\sigma)=\bigoplus_{\kappa \in \hat{R}} \pi_{\kappa}
$$

Moreover, replacing $A_{i}$ with $-A_{i}$ if necessary, we may assume that $\pi_{1}$ is $\chi$-generic. Here $\chi$ is a fixed non-degenerate character of the unipotent radical of the standard Borel subgroup of $G$.

Similarly, we write $I^{\prime}(\sigma, \lambda)=\operatorname{Ind}_{P^{\prime}}^{G^{\prime}}\left(\sigma||^{\lambda}\right)$ and $I^{\prime}(\sigma)=I^{\prime}(\sigma, 0)$. Here we regard $P^{\prime}=L^{\prime} U^{\prime}$ with $L^{\prime}=L$ and $U^{\prime}=U$ as a parabolic subgroup of $G^{\prime}$. We also regard $W^{\prime}=W$ as the Weyl group of $G^{\prime}, w_{i}^{\prime}=w_{i}$ as an element of $G^{\prime}, r_{i}^{\prime}=r_{i}$ as an element of $W^{\prime}, R^{\prime}=R$ as a subgroup of $W^{\prime}$, and $\chi$ as a non-degenerate character of the unipotent radical of the standard Borel subgroup of $G^{\prime}$. For each $\kappa^{\prime} \in \hat{R}^{\prime}$, let

$$
\pi_{\kappa^{\prime}}^{\prime}=\left\{f^{\prime} \in I^{\prime}(\sigma) \mid \mathcal{N}\left(r^{\prime}, \sigma\right) f^{\prime}=\kappa^{\prime}\left(r^{\prime}\right) f^{\prime} \text { for all } r^{\prime} \in R^{\prime}\right\} .
$$

Here we regard $\mathcal{N}\left(r^{\prime}, \sigma\right)$ as a self-intertwining operator of $I^{\prime}(\sigma)$. Then $\pi_{\kappa^{\prime}}^{\prime}$ is irreducible, $\pi_{1}^{\prime}$ is $\chi$-generic, and

$$
I^{\prime}(\sigma)=\bigoplus_{\kappa^{\prime} \in \hat{R}^{\prime}} \pi_{\kappa^{\prime}}^{\prime}
$$

\section{The main theorem}

Let $\pi$ (respectively $\pi^{\prime}$ ) be an irreducible admissible representation of $G$ (respectively $G^{\prime}$ ). We write $\theta(\pi)=\pi^{\prime}$ if

$$
\operatorname{Hom}_{G \times G^{\prime}}\left(\omega, \tilde{\pi} \otimes \pi^{\prime}\right) \neq 0 .
$$

Waldspurger [Wal90] showed that $\theta(\pi)$ is uniquely determined if it exists.

Let $\kappa \in \hat{R}$. By Lemma 1.1, we can define $\theta(\kappa) \in \hat{R}^{\prime}$ by

$$
\theta(\kappa)\left(r_{i}^{\prime}\right)=\kappa\left(r_{i}\right) \cdot \epsilon\left(1 / 2, \sigma_{i}, \psi\right) \omega_{\sigma_{i}}(\delta)^{-1}
$$

for $i \in \mathfrak{I}$. Then our main result is as follows.

Theorem 4.1. For $\kappa \in \hat{R}$,

$$
\theta\left(\pi_{\kappa}\right)=\pi_{\theta(\kappa)}^{\prime}
$$

We will give the proof in the next two sections. 


\section{LOCAL THETA CORRESPONDENCE AND $R$-GROUPS}

Remark 4.2. Even if $p=2$, we can prove that

$$
\operatorname{Hom}_{G \times G^{\prime}}\left(\omega, \tilde{\pi}_{\kappa} \otimes \pi_{\theta(\kappa)}^{\prime}\right) \neq 0 .
$$

\section{An equivariant map}

Let $\pi$ (respectively $\pi^{\prime}$ ) be an admissible representation of $G$ (respectively $G^{\prime}$ ). A map $T: \omega \otimes \pi \rightarrow \pi^{\prime}$ is said to be $\left(G \times G^{\prime}\right)$-equivariant if

$$
T\left(\omega\left(g, g^{\prime}\right) \Phi, \pi(g) f\right)=\pi^{\prime}\left(g^{\prime}\right) T(\Phi, f)
$$

for all $g \in G, g^{\prime} \in G^{\prime}, \Phi \in \mathcal{S}$, and $f \in \pi$. Let $\operatorname{Hom}_{G \times G^{\prime}}\left(\omega \otimes \pi, \pi^{\prime}\right)$ denote the space of $\left(G \times G^{\prime}\right)$ equivariant maps $T: \omega \otimes \pi \rightarrow \pi^{\prime}$. Then

$$
\operatorname{Hom}_{G \times G^{\prime}}\left(\omega \otimes \pi, \pi^{\prime}\right) \simeq \operatorname{Hom}_{G \times G^{\prime}}\left(\omega, \tilde{\pi} \otimes \pi^{\prime}\right) .
$$

In this section, we construct explicitly an element of

$$
\operatorname{Hom}_{G \times G^{\prime}}\left(\omega \otimes I(\sigma, \lambda), I^{\prime}(\sigma, \lambda)\right),
$$

and study its properties.

For $\Phi \in \mathcal{S}$, define a function $F_{\Phi}$ on $G \times G^{\prime}$ by

$$
F_{\Phi}\left(g, g^{\prime}\right)=\int_{Z_{\mathbf{n}}} \omega\left(g, g^{\prime}\right) \Phi\left(\begin{array}{l}
z \\
0
\end{array}\right) \psi(\operatorname{tr}(z)) d z .
$$

Then $F_{\Phi}$ is left $\left(U \times U^{\prime}\right)$-invariant and satisfies

$$
F_{\Phi}\left(a g, a g^{\prime}\right)=|a|^{2 \rho} F_{\Phi}\left(g, g^{\prime}\right)
$$

for $a \in L_{\mathbf{n}}$. Here we regard $a$ as an element of $L=L^{\prime}$. Let $\tilde{\sigma}$ be the contragredient representation of $\sigma$ and $\tilde{\mathcal{V}}$ the space of $\tilde{\sigma}$. For $g^{\prime} \in G^{\prime}, \Phi \in \mathcal{S}$, a holomorphic section $f^{(\lambda)}$ of $I(\sigma, \lambda)$, and $\tilde{v} \in \tilde{\mathcal{V}}$, put

$$
Z\left(g^{\prime}, \Phi, f^{(\lambda)}, \tilde{v}\right)=\prod_{i=1}^{t} L\left(\lambda_{i}+1 / 2, \sigma_{i}\right)^{-1} \int_{U \backslash G} F_{\Phi}\left(g, g^{\prime}\right)\left\langle f^{(\lambda)}(g), \tilde{v}\right\rangle d g .
$$

Here $\langle\rangle:, \mathcal{V} \times \tilde{\mathcal{V}} \rightarrow \mathbb{C}$ is the natural pairing.

Lemma 5.1. If $\operatorname{Re}\left(\lambda_{i}\right) \gg 0$ for all $i$, then $Z\left(g^{\prime}, \Phi, f^{(\lambda)}, \tilde{v}\right)$ is absolutely convergent. Moreover it extends to a holomorphic function of $\lambda$ on $\mathbb{C}^{t}$. In particular, for fixed $g^{\prime}, \Phi, \tilde{v}$, and $\lambda_{0} \in \mathbb{C}^{t}$, the value of $Z\left(g^{\prime}, \Phi, f^{(\lambda)}, \tilde{v}\right)$ at $\lambda=\lambda_{0}$ depends only on $f^{\left(\lambda_{0}\right)}$.

Proof. For each $\Phi \in \mathcal{S}$, define $\Psi(\Phi) \in \mathcal{S}\left(M_{n_{1}}(E) \oplus \cdots \oplus M_{n_{t}}(E)\right)$ by

$$
\Psi\left(x_{1}, \ldots, x_{t} ; \Phi\right)=\int_{Z_{\mathbf{n}}} \Phi\left(\begin{array}{l}
z \\
0
\end{array}\right) \psi(\operatorname{tr}(x z)) d z,
$$

where $x_{i} \in M_{n_{i}}(E)$ for $1 \leqslant i \leqslant t$ and $x=\operatorname{diag}\left(x_{1}, \ldots, x_{t}\right) \in M_{n}(E)$. Then

$$
F_{\Phi}\left(a g, g^{\prime}\right)=\prod_{i=1}^{t}\left|a_{i}\right|^{n_{i}+\cdots+n_{t}} \Psi\left(a_{1}, \ldots, a_{t} ; \omega\left(g, g^{\prime}\right) \Phi\right)
$$

for $a=\operatorname{diag}\left(a_{1}, \ldots, a_{t}\right) \in L_{\mathbf{n}}$. Hence

$$
\begin{gathered}
\prod_{i=1}^{t} L\left(\lambda_{i}+1 / 2, \sigma_{i}\right) Z\left(g^{\prime}, \Phi, f^{(\lambda)}, \tilde{v}\right)=\int_{L \times K} F_{\Phi}\left(a k, g^{\prime}\right)\left\langle\sigma(a) f^{(\lambda)}(k), \tilde{v}\right\rangle|a|^{\lambda-\rho} d a d k \\
\quad=\int_{L_{\mathbf{n}}} \int_{K} \Psi\left(a_{1}, \ldots, a_{t} ; \omega\left(k, g^{\prime}\right) \Phi\right)\left\langle\sigma(a) f^{(\lambda)}(k), \tilde{v}\right\rangle \prod_{i=1}^{t}\left|a_{i}\right|^{\lambda_{i}+n_{i} / 2} d k d a
\end{gathered}
$$

and this concludes the proof. 


\section{A. ICHINO}

Let $g^{\prime} \in G^{\prime}$ and $\Phi \in \mathcal{S}$. Let $f^{(\lambda)}$ be a holomorphic section of $I(\sigma, \lambda)$. Then we see that there exists an element $T\left(g^{\prime} ; \Phi, f^{(\lambda)}\right)$ of $\mathcal{V}$ such that

$$
\left\langle T\left(g^{\prime} ; \Phi, f^{(\lambda)}\right), \tilde{v}\right\rangle=Z\left(g^{\prime}, \Phi, f^{(\lambda)}, \tilde{v}\right)
$$

for all $\tilde{v} \in \tilde{\mathcal{V}}$. Moreover it satisfies

$$
T\left(p^{\prime} g^{\prime} ; \Phi, f^{(\lambda)}\right)=|a|^{\lambda+\rho} \sigma(a) T\left(g^{\prime} ; \Phi, f^{(\lambda)}\right)
$$

for $p^{\prime}=a u^{\prime} \in P^{\prime}$ with $a \in L^{\prime}$ and $u^{\prime} \in U^{\prime}$, hence defines a holomorphic section $T\left(\Phi, f^{(\lambda)}\right)$ of $I^{\prime}(\sigma, \lambda)$. Thus we obtain a $\left(G \times G^{\prime}\right)$-equivariant map

$$
T: \omega \otimes I(\sigma, \lambda) \longrightarrow I^{\prime}(\sigma, \lambda)
$$

Fix $d \leqslant t$. Let $\mathbf{l}=\left(n_{1}, \ldots, n_{d}\right)$ and $\mathbf{m}=\left(n_{d+1}, \ldots, n_{t}\right)$ be partitions of $l=n_{1}+\cdots+n_{d}$ and $m=n_{d+1}+\cdots+n_{t}$, respectively. For $\Phi \in \mathcal{S}$, define another function $\hat{F}_{\Phi}$ on $G \times G^{\prime}$ by

$$
\hat{F}_{\Phi}\left(g, g^{\prime}\right)=\int_{u \in U_{1}} \int_{z \in Z_{\mathbf{m}}} \int_{v \in M_{l, m}(E)} \omega\left(g, g^{\prime}\right) \Phi\left(\begin{array}{cc}
u & v \\
0 & z \\
0 & 0 \\
0 & 0
\end{array}\right) \psi(\operatorname{tr}(z)) d v d z d u
$$

Then $\hat{F}_{\Phi}$ is also left $\left(U \times U^{\prime}\right)$-invariant.

Lemma 5.2. If $\operatorname{Re}\left(\lambda_{i}\right) \ll 0$ for all $i \leqslant d$ and $\operatorname{Re}\left(\lambda_{j}\right) \gg 0$ for all $j>d$, then

$$
\begin{aligned}
\left\langle T\left(g^{\prime} ; \Phi, f^{(\lambda)}\right), \tilde{v}\right\rangle= & \prod_{i=1}^{d} \omega_{\sigma_{i}}(-1) \epsilon\left(\lambda_{i}+1 / 2, \sigma_{i}, \psi\right)^{-1} L\left(-\lambda_{i}+1 / 2, \tilde{\sigma}_{i}\right)^{-1} \\
& \times \prod_{j=d+1}^{t} L\left(\lambda_{j}+1 / 2, \sigma_{j}\right)^{-1} \int_{U \backslash G} \hat{F}_{\Phi}\left(g, g^{\prime}\right)\left\langle f^{(\lambda)}(g), \tilde{v}\right\rangle d g .
\end{aligned}
$$

Proof. Let $\Psi \in \mathcal{S}\left(M_{n_{1}}(E) \oplus \cdots \oplus M_{n_{t}}(E)\right)$. We define the partial Fourier transform $\hat{\Psi} \in \mathcal{S}\left(M_{n_{1}}(E) \oplus\right.$ $\left.\cdots \oplus M_{n_{t}}(E)\right)$ of $\Psi$ by

$$
\hat{\Psi}\left(x_{1}, \ldots, x_{t}\right)=\int_{M_{n_{1}}(E) \oplus \cdots \oplus M_{n_{d}}(E)} \Psi\left(y_{1}, \ldots, y_{d}, x_{d+1}, \ldots, x_{t}\right) \psi\left(\operatorname{tr}\left(x^{\prime} y\right)\right) d y_{1} \cdots d y_{d},
$$

where $x^{\prime}=\operatorname{diag}\left(x_{1}, \ldots, x_{d}\right) \in M_{l}(E)$ and $y=\operatorname{diag}\left(y_{1}, \ldots, y_{d}\right) \in M_{l}(E)$. For $1 \leqslant i \leqslant t$, let $\phi_{i}$ be a matrix coefficient of $\sigma_{i}$. If $\left|\operatorname{Re}\left(\lambda_{i}\right)\right|<1 / 2$ for all $i \leqslant d$ and $\operatorname{Re}\left(\lambda_{j}\right)>-1 / 2$ for all $j>d$, then we have

$$
\begin{aligned}
\prod_{i=1}^{d} L( & \left.\lambda_{i}+1 / 2, \sigma_{i}\right)^{-1} \int_{L_{\mathbf{n}}} \Psi\left(a_{1}, \ldots, a_{t}\right) \prod_{i=1}^{t} \phi_{i}\left(a_{i}\right)\left|a_{i}\right|^{\lambda_{i}+n_{i} / 2} d a \\
= & \prod_{i=1}^{d} \epsilon\left(\lambda_{i}+1 / 2, \sigma_{i}, \psi\right)^{-1} L\left(-\lambda_{i}+1 / 2, \tilde{\sigma}_{i}\right)^{-1} \\
& \times \int_{L_{\mathbf{n}}} \hat{\Psi}\left(a_{1}, \ldots, a_{t}\right) \prod_{i=1}^{d} \check{\phi}_{i}\left(a_{i}\right)\left|a_{i}\right|^{-\lambda_{i}+n_{i} / 2} \prod_{j=d+1}^{t} \phi_{j}\left(a_{j}\right)\left|a_{j}\right|^{\lambda_{j}+n_{j} / 2} d a
\end{aligned}
$$

by the functional equation (1.1). 


\section{LOCAL THETA CORRESPONDENCE AND $R$-GROUPS}

We now take $\Psi=\Psi(\Phi)$ as in the proof of Lemma 5.1. Then

$$
\begin{aligned}
\hat{\Psi}\left(x_{1}, \ldots, x_{t} ; \Phi\right)= & \int_{M_{n_{1}}(E) \oplus \cdots \oplus M_{n_{d}}(E)} \int_{z^{\prime} \in Z_{\mathbf{l}}} \int_{z^{\prime \prime} \in Z_{\mathbf{m}}} \int_{v \in M_{l, m}(E)} \Phi\left(\begin{array}{cc}
z^{\prime} & v \\
0 & z^{\prime \prime} \\
0 & 0 \\
0 & 0
\end{array}\right) \\
& \times \psi\left(\operatorname{tr}\left(y z^{\prime}\right)+\operatorname{tr}\left(x^{\prime \prime} z^{\prime \prime}\right)\right) \psi\left(\operatorname{tr}\left(x^{\prime} y\right)\right) d v d z^{\prime \prime} d z^{\prime} d y_{1} \cdots d y_{d},
\end{aligned}
$$

where $x^{\prime \prime}=\operatorname{diag}\left(x_{d+1}, \ldots, x_{t}\right) \in M_{m}(E)$. By the Fourier inversion formula, this integral is equal to

$$
\int_{u \in U_{\mathbf{l}}} \int_{z^{\prime \prime} \in Z_{\mathbf{m}}} \int_{v \in M_{l, m}(E)} \Phi\left(\begin{array}{cc}
z^{\prime}\left(u, x^{\prime}\right) & v \\
0 & z^{\prime \prime} \\
0 & 0 \\
0 & 0
\end{array}\right) \psi\left(\operatorname{tr}\left(x^{\prime \prime} z^{\prime \prime}\right)\right) d v d z^{\prime \prime} d u
$$

where $z^{\prime}\left(u, x^{\prime}\right)=\left(z_{i j}^{\prime}\right) \in Z_{1}$ with $z_{i j}^{\prime}=u_{i j}$ for $1 \leqslant i<j \leqslant d$ and $z_{i i}^{\prime}=-x_{i}$ for $1 \leqslant i \leqslant d$. Hence

$$
\hat{\Psi}\left(-a_{1}^{-1}, \ldots,-a_{d}^{-1}, a_{d+1}, \ldots, a_{t} ; \Phi\right)=\prod_{i=1}^{d}\left|a_{i}\right|^{-n_{i+1}-\cdots-n_{t}} \prod_{j=d+1}^{t}\left|a_{j}\right|^{-n_{j}-\cdots-n_{t}} \hat{F}_{\Phi}(a, 1)
$$

for $a=\operatorname{diag}\left(a_{1}, \ldots, a_{t}\right) \in L_{\mathbf{n}}$. Therefore

$$
\begin{gathered}
\int_{L_{\mathbf{n}}} \hat{\Psi}\left(a_{1}, \ldots, a_{t} ; \Phi\right) \prod_{i=1}^{d} \check{\phi}_{i}\left(a_{i}\right)\left|a_{i}\right|^{-\lambda_{i}+n_{i} / 2} \prod_{j=d+1}^{t} \phi_{j}\left(a_{j}\right)\left|a_{j}\right|^{\lambda_{j}+n_{j} / 2} d a \\
=\int_{L} \hat{F}_{\Phi}(a, 1) \prod_{i=1}^{d} \omega_{\sigma_{i}}(-1) \phi_{i}\left(a_{i}\right) \prod_{j=d+1}^{t} \phi_{j}\left(a_{j}\right)|a|^{\lambda-\rho} d a
\end{gathered}
$$

and this concludes the proof.

Let $H=G_{m}$ and $H^{\prime}=G_{m}^{\prime}$. We define embeddings $H \hookrightarrow G$ and $H^{\prime} \hookrightarrow G^{\prime}$ by

$$
\left(\begin{array}{cc}
A & B \\
C & D
\end{array}\right) \longmapsto\left(\begin{array}{cc|cc}
\mathbf{1}_{l} & 0 & 0 & 0 \\
0 & A & 0 & B \\
\hline 0 & 0 & \mathbf{1}_{l} & 0 \\
0 & C & 0 & D
\end{array}\right)
$$

We also regard $L_{\mathbf{l}}$ and $L_{\mathbf{m}}$ as subgroups of $L_{\mathbf{n}}$. Let $\omega_{H}$ be the Weil representation of $H \times H^{\prime}$ on $\mathcal{S}_{H}=\mathcal{S}\left(V_{m}^{m}\right)=\mathcal{S}\left(M_{2 m, m}(E)\right)$ as in $\S 2$. Let $\sigma^{\prime}=\sigma_{1} \otimes \cdots \otimes \sigma_{d}$ and $\sigma_{H}=\sigma_{d+1} \otimes \cdots \otimes \sigma_{t}$. For $\lambda=\left(\lambda_{1}, \ldots, \lambda_{t}\right) \in \mathbb{C}^{t}$, put $\lambda_{H}=\left(\lambda_{d+1}, \ldots, \lambda_{t}\right) \in \mathbb{C}^{t-d}$. Then we have an $\left(H \times H^{\prime}\right)$-equivariant map

$$
T_{H}: \omega_{H} \otimes I\left(\sigma_{H}, \lambda_{H}\right) \longrightarrow I^{\prime}\left(\sigma_{H}, \lambda_{H}\right)
$$

as in (5.1). Define an $\left(H \times H^{\prime}\right)$-equivariant map

$$
\begin{aligned}
& \mathcal{S} \longrightarrow \mathcal{S}_{H} \\
& \Phi \longmapsto \Phi_{H}
\end{aligned}
$$

by

$$
\Phi_{H}\left(\begin{array}{l}
x \\
y
\end{array}\right)=\int_{z^{\prime} \in Z_{1}} \int_{v \in M_{l, m}(E)} \Phi\left(\begin{array}{cc}
z^{\prime} & v \\
0 & x \\
0 & 0 \\
0 & y
\end{array}\right) \psi\left(\operatorname{tr}\left(z^{\prime}\right)\right) d v d z^{\prime}
$$




\section{A. ICHINO}

Let $\tilde{\mathcal{V}}^{\prime}$ and $\tilde{\mathcal{V}}_{H}$ denote the space of $\tilde{\sigma}^{\prime}$ and $\tilde{\sigma}_{H}$, respectively. For a holomorphic section $f^{(\lambda)}$ of $I(\sigma, \lambda)$, $g \in G$, and $\tilde{v}^{\prime} \in \tilde{\mathcal{V}}^{\prime}$, define a holomorphic section $f_{H}^{(\lambda)}\left(g, \tilde{v}^{\prime}\right)$ of $I\left(\sigma_{H}, \lambda_{H}\right)$ by

$$
f_{H}^{(\lambda)}\left(h ; g, \tilde{v}^{\prime}\right)=\left[\tilde{\mathcal{V}}_{H} \ni \tilde{u} \longmapsto\left\langle f^{(\lambda)}(h g), \tilde{v}^{\prime} \otimes \tilde{u}\right\rangle\right]
$$

for $h \in H$. The following lemma reduces an intertwining property of $T$ with respect to $H$ and $H^{\prime}$ to that of $T_{H}$.

Lemma 5.3. If $\operatorname{Re}\left(\lambda_{i}\right) \gg 0$ for all $i \leqslant d$, then

$$
\begin{aligned}
& \operatorname{vol}\left(K_{H}\right) \prod_{i=1}^{d} L\left(\lambda_{i}+1 / 2, \sigma_{i}\right)\left\langle T\left(h^{\prime} ; \Phi, f^{(\lambda)}\right), \tilde{v}^{\prime} \otimes \tilde{u}\right\rangle \\
& \quad=\int_{L_{1} \times K}\left\langle T_{H}\left(h^{\prime} ;\left[\omega\left(a^{\prime} k, 1\right) \Phi\right]_{H}, f_{H}^{(\lambda)}\left(k, \tilde{\sigma}^{\prime}\left(a^{\prime-1}\right) \tilde{v}^{\prime}\right)\right), \tilde{u}\right\rangle\left|a^{\prime}\right|^{\lambda-\rho} d a^{\prime} d k
\end{aligned}
$$

for $h^{\prime} \in H^{\prime}$. Here $K_{H}=H \cap G L_{2 m}\left(\mathfrak{o}_{E}\right)$.

Proof. First, observe that the right-hand side is absolutely convergent. Hence we may assume that $\operatorname{Re}\left(\lambda_{i}\right) \gg 0$ for all $i \leqslant t$. For $a=\operatorname{diag}\left(a_{1}, \ldots, a_{t}\right) \in L_{\mathbf{n}}$, we write $a^{\prime}=\operatorname{diag}\left(a_{1}, \ldots, a_{d}\right) \in L_{\mathbf{l}}$ and $a^{\prime \prime}=\operatorname{diag}\left(a_{d+1}, \ldots, a_{t}\right) \in L_{\mathbf{m}}$. Then

$$
\begin{aligned}
\operatorname{vol}\left(K_{H}\right) & \int_{U \backslash G} F_{\Phi}\left(g, h^{\prime}\right)\left\langle f^{(\lambda)}(g), \tilde{v}^{\prime} \otimes \tilde{u}\right\rangle d g \\
= & \operatorname{vol}\left(K_{H}\right) \int_{L_{\mathbf{n}} \times K} \int_{z \in Z_{\mathbf{n}}} \omega\left(a k, h^{\prime}\right) \Phi\left(\begin{array}{c}
z \\
0
\end{array}\right) \psi(\operatorname{tr}(z))\left\langle\sigma(a) f^{(\lambda)}(k), \tilde{v}^{\prime} \otimes \tilde{u}\right\rangle|a|^{\lambda-\rho} d z d a d k \\
= & \int_{k_{0} \in K_{H}} \int_{L_{\mathbf{1}} \times L_{\mathbf{m}} \times K} \int_{z^{\prime} \in Z_{\mathbf{l}}} \int_{z^{\prime \prime} \in Z_{\mathbf{m}}} \int_{v \in M_{l, m}(E)} \omega\left(a^{\prime} a^{\prime \prime} k_{0} k, h^{\prime}\right) \Phi\left(\begin{array}{cc}
z^{\prime} & v \\
0 & z^{\prime \prime} \\
0 & 0 \\
0 & 0
\end{array}\right) \\
& \times \psi\left(\operatorname{tr}\left(z^{\prime}\right)+\operatorname{tr}\left(z^{\prime \prime}\right)\right)\left\langle\sigma\left(a^{\prime \prime}\right) f^{(\lambda)}\left(k_{0} k\right), \tilde{\sigma}^{\prime}\left(a^{\prime-1}\right) \tilde{v}^{\prime} \otimes \tilde{u}\right\rangle\left|a^{\prime} a^{\prime \prime}\right|^{\lambda-\rho} d v d z^{\prime \prime} d z^{\prime} d a^{\prime} d a^{\prime \prime} d k d k_{0} \\
= & \int_{L_{\mathbf{1}} \times K} \int_{L_{\mathbf{m}} \times K_{H}} \int_{Z_{\mathbf{m}}} \omega_{H}\left(a^{\prime \prime} k_{0}, h^{\prime}\right)\left[\omega\left(a^{\prime} k, 1\right) \Phi\right]_{H}\left(\begin{array}{c}
z^{\prime \prime} \\
0
\end{array}\right) \psi\left(\operatorname{tr}\left(z^{\prime \prime}\right)\right) \\
& \times\left\langle\sigma_{H}\left(a^{\prime \prime}\right) f_{H}^{(\lambda)}\left(k_{0} ; k, \tilde{\sigma}^{\prime}\left(a^{\prime-1}\right) \tilde{v}^{\prime}\right), \tilde{u}\right\rangle\left|a^{\prime} a^{\prime \prime}\right|^{\lambda-\rho} d z^{\prime \prime} d a^{\prime \prime} d k_{0} d a^{\prime} d k \\
= & \int_{L_{1} \times K} \int_{L_{\mathbf{m}} \times K_{H}} F_{\left[\omega\left(a^{\prime} k, 1\right) \Phi\right]_{H}}\left(a^{\prime \prime} k_{0}, h^{\prime}\right) \\
& \times\left\langle\sigma_{H}\left(a^{\prime \prime}\right) f_{H}^{(\lambda)}\left(k_{0} ; k, \tilde{\sigma}^{\prime}\left(a^{\prime-1}\right) \tilde{v}^{\prime}\right), \tilde{u}\right\rangle\left|a^{\prime} a^{\prime \prime}\right|^{\lambda-\rho} d a^{\prime \prime} d k_{0} d a^{\prime} d k .
\end{aligned}
$$

Lemma 5.4. Let $\lambda_{0} \in \mathbb{C}^{t}$ and assume that

$$
\prod_{i=1}^{t} L\left(-\lambda_{i}+1 / 2, \tilde{\sigma}_{i}\right)
$$

is holomorphic at $\lambda=\lambda_{0}$. Let $f^{(\lambda)}$ be a holomorphic section of $I(\sigma, \lambda)$ such that $f^{\left(\lambda_{0}\right)} \neq 0$. Then there exists $\Phi \in \mathcal{S}$ such that

$$
T\left(\Phi, f^{\left(\lambda_{0}\right)}\right) \neq 0 .
$$

In particular, for a subrepresentation $\pi$ of $I\left(\sigma, \lambda_{0}\right)$,

$$
\left.T\right|_{\omega \otimes \pi}: \omega \otimes \pi \longrightarrow I^{\prime}\left(\sigma, \lambda_{0}\right)
$$

is a non-zero $\left(G \times G^{\prime}\right)$-equivariant map. 


\section{LOCAL THETA CORRESPONDENCE AND $R$-GROUPS}

Proof. We may assume that $f^{(\lambda)}$ is a standard section, i.e., its restriction to $K$ is independent of $\lambda$. Put $d=t$ and assume that $\operatorname{Re}\left(\lambda_{i}\right) \ll 0$ for all $i$. By Lemma 5.2, it suffices to show that there exist $\Phi \in \mathcal{S}$ and $\tilde{v} \in \tilde{\mathcal{V}}$ such that

$$
\int_{U \backslash G} \hat{F}_{\Phi}(g, 1)\left\langle f^{(\lambda)}(g), \tilde{v}\right\rangle d g
$$

is non-zero and independent of $\lambda$.

Let

and

$$
N=\left\{\left(\begin{array}{cc}
\mathbf{1}_{n} & b \\
\mathbf{0}_{n} & \mathbf{1}_{n}
\end{array}\right) \in G \mid b \in X_{n}\right\}
$$

$$
x_{0}=\left(\begin{array}{l}
\mathbf{1}_{n} \\
\mathbf{0}_{n}
\end{array}\right) \in V^{n}=M_{2 n, n}(E)
$$

Since

$$
\hat{F}_{\Phi}(g, 1)=\int_{U_{\mathbf{n}}} \omega(g, 1) \Phi\left(\begin{array}{l}
u \\
0
\end{array}\right) d u=\int_{N \backslash U} \omega(u g, 1) \Phi\left(x_{0}\right) d u
$$

we have

$$
(5.2)=\int_{N \backslash G} \omega(g, 1) \Phi\left(x_{0}\right)\left\langle f^{(\lambda)}(g), \tilde{v}\right\rangle d g .
$$

Take $\tilde{v} \in \tilde{\mathcal{V}}$ so that $\left\langle f^{(\lambda)}(g), \tilde{v}\right\rangle \neq 0$ for some $g \in G$. We define a non-zero smooth function $\varphi$ on $K$ by $\varphi(k)=\left\langle f^{(\lambda)}(k), \tilde{v}\right\rangle$ for $k \in K$. Then $\varphi$ is left $(N \cap K)$-invariant and does not depend on $\lambda$. Since

$$
\begin{aligned}
N \backslash G & \stackrel{\sim}{\longrightarrow} G \cdot x_{0} \\
g & \longmapsto g^{-1} x_{0}
\end{aligned}
$$

is a homeomorphism and $G \cdot x_{0}$ is locally closed in $V^{n}$, there exists $\Phi \in \mathcal{S}$ such that $\operatorname{supp} \Phi \cap G \cdot x_{0}=$ $K \cdot x_{0}$ and $\Phi\left(k^{-1} x_{0}\right)=\overline{\varphi(k)}$ for all $k \in K$. Then

$$
(5.2)=\int_{N \backslash N K} \Phi\left(g^{-1} x_{0}\right)\left\langle f^{(\lambda)}(g), \tilde{v}\right\rangle d g=\int_{(N \cap K) \backslash K}|\varphi(k)|^{2} d k \neq 0 .
$$

\section{Compatibility with intertwining operators}

In this section, we complete the proof of Theorem 4.1. A key step is to show that the $\left(G \times G^{\prime}\right)$ equivariant map (5.1) is compatible with the action of intertwining operators.

Lemma 6.1. For $\varphi \in \mathcal{S}\left(M_{l}(E)\right)$,

$$
\int_{x^{\prime} \in X_{l}} \int_{v \in M_{l}(E)} \varphi(v) \psi\left(\operatorname{tr}\left({ }^{t} \bar{v} x^{\prime}\right)\right) d v d x^{\prime}=|\delta|^{l^{2} / 2} \int_{x \in X_{l}} \varphi(\delta x) d x .
$$

Proof. We write $v=\delta y+y^{\prime}$ with $y, y^{\prime} \in X_{l}$. Then the left-hand side is equal to

$$
|\delta|^{l^{2} / 2} \int_{x^{\prime} \in X_{l}} \int_{y^{\prime} \in X_{l}} \int_{y \in X_{l}} \varphi\left(\delta y+y^{\prime}\right) \psi\left(\operatorname{tr}\left({ }^{(} \bar{y}^{\prime} x^{\prime}\right)\right) d y d y^{\prime} d x^{\prime}
$$

Hence the lemma follows from the Fourier inversion formula.

Let $d=1$, i.e., $l=n_{1}, m=n-n_{1}, \mathbf{l}=\left(n_{1}\right)$, and $\mathbf{m}=\left(n_{2}, \ldots, n_{t}\right)$. For $x \in X_{l}$ and $y, b \in M_{l, m}(E)$, define elements $u(x, y)$ and $u(b)$ of $U$ by

$$
u(x, y)=\left(\begin{array}{cc|cc}
\mathbf{1}_{l} & 0 & x & y \\
0 & \mathbf{1}_{m} & { }^{t} \bar{y} & 0 \\
\hline 0 & 0 & \mathbf{1}_{l} & 0 \\
0 & 0 & 0 & \mathbf{1}_{m}
\end{array}\right), \quad u(b)=\left(\begin{array}{cc|cc}
\mathbf{1}_{l} & b & 0 & 0 \\
0 & \mathbf{1}_{m} & 0 & 0 \\
\hline 0 & 0 & \mathbf{1}_{l} & 0 \\
0 & 0 & -{ }^{t} \bar{b} & \mathbf{1}_{m}
\end{array}\right) .
$$




\section{A. ICHINO}

In the same way, define elements $u^{\prime}\left(x^{\prime}, y^{\prime}\right)$ and $u^{\prime}\left(b^{\prime}\right)$ of $U^{\prime}$ for $x^{\prime} \in X_{l}$ and $y^{\prime}, b^{\prime} \in M_{l, m}(E)$. We put

$$
w=\left(\begin{array}{cc|cc}
0 & 0 & \mathbf{1}_{l} & 0 \\
0 & \mathbf{1}_{m} & 0 & 0 \\
\hline-\mathbf{1}_{l} & 0 & 0 & 0 \\
0 & 0 & 0 & \mathbf{1}_{m}
\end{array}\right) \in G
$$

and regard $w^{\prime}=w$ as an element of $G^{\prime}$.

Proposition 6.2.

$$
M\left(w^{\prime}, \sigma, \lambda\right) T\left(\Phi, f^{(\lambda)}\right)=|\delta|^{n_{1} \lambda_{1}} \omega_{\sigma_{1}}(-\delta) \epsilon\left(-\lambda_{1}+1 / 2,{ }^{t} \bar{\sigma}_{1}^{-1}, \psi\right) T\left(\Phi, M(w, \sigma, \lambda) f^{(\lambda)}\right) .
$$

Proof. We may assume that $\operatorname{Re}\left(\lambda_{1}\right) \gg \cdots \gg \operatorname{Re}\left(\lambda_{t}\right) \gg 0$. We put

$$
a_{\delta}=\left(\begin{array}{cc}
\delta \mathbf{1}_{l} & 0 \\
0 & \mathbf{1}_{m}
\end{array}\right) \in L_{\mathbf{n}}
$$

and regard $a_{\delta}$ as an element of $L$. Let $\tilde{v} \in \tilde{\mathcal{V}}$. Then

$$
\begin{aligned}
\int_{U \backslash G} F_{\Phi}\left(a_{\delta} g, g^{\prime}\right)\left\langle f^{(\lambda)}(g), \tilde{v}\right\rangle d g & =\left|a_{\delta}\right|^{2 \rho} \int_{U \backslash G} F_{\Phi}\left(g, g^{\prime}\right)\left\langle f^{(\lambda)}\left(a_{\delta}^{-1} g\right), \tilde{v}\right\rangle d g \\
& =\left|a_{\delta}\right|^{-\lambda+\rho} \omega_{\sigma_{1}}(\delta)^{-1} \int_{U \backslash G} F_{\Phi}\left(g, g^{\prime}\right)\left\langle f^{(\lambda)}(g), \tilde{v}\right\rangle d g
\end{aligned}
$$

Hence

$$
\begin{aligned}
\prod_{i=1}^{t} L & \left(\lambda_{i}+1 / 2, \sigma_{i}\right)\left\langle M\left(w^{\prime}, \sigma, \lambda\right) T\left(g^{\prime} ; \Phi, f^{(\lambda)}\right), \tilde{v}\right\rangle \\
& =\prod_{i=1}^{t} L\left(\lambda_{i}+1 / 2, \sigma_{i}\right) \int_{\left(U^{\prime} \cap w^{\prime} U^{\prime} w^{\prime-1}\right) \backslash U^{\prime}}\left\langle T\left(w^{\prime-1} u^{\prime} g^{\prime} ; \Phi, f^{(\lambda)}\right), \tilde{v}\right\rangle d u^{\prime} \\
& =\int_{\left(U^{\prime} \cap w^{\prime} U^{\prime} w^{\prime-1}\right) \backslash U^{\prime}} \int_{U \backslash G} F_{\Phi}\left(g, w^{\prime-1} u^{\prime} g^{\prime}\right)\left\langle f^{(\lambda)}(g), \tilde{v}\right\rangle d g d u^{\prime} \\
& =\left|a_{\delta}\right|^{\lambda-\rho} \omega_{\sigma_{1}}(\delta) \int_{U \backslash G} \int_{\left(U^{\prime} \cap w^{\prime} U^{\prime} w^{\prime-1}\right) \backslash U^{\prime}} F_{\Phi}\left(a_{\delta} g, w^{\prime-1} u^{\prime} g^{\prime}\right)\left\langle f^{(\lambda)}(g), \tilde{v}\right\rangle d u^{\prime} d g .
\end{aligned}
$$

On the other hand, by Lemma 5.2, we have

$$
\begin{aligned}
\omega_{\sigma_{1}}(- & 1) \epsilon\left(-\lambda_{1}+1 / 2,{ }^{t} \bar{\sigma}_{1}^{-1}, \psi\right) L\left(\lambda_{1}+1 / 2, \bar{\sigma}_{1}\right) \prod_{j=2}^{t} L\left(\lambda_{j}+1 / 2, \sigma_{j}\right)\left\langle T\left(g^{\prime} ; \Phi, M(w, \sigma, \lambda) f^{(\lambda)}\right), \tilde{v}\right\rangle \\
& =\int_{U \backslash G} \hat{F}_{\Phi}\left(g, g^{\prime}\right)\left\langle M(w, \sigma, \lambda) f^{(\lambda)}(g), \tilde{v}\right\rangle d g \\
& =\int_{U \backslash G} \int_{\left(U \cap w U w^{-1}\right) \backslash U} \hat{F}_{\Phi}\left(g, g^{\prime}\right)\left\langle f^{(\lambda)}\left(w^{-1} u g\right), \tilde{v}\right\rangle d u d g \\
& =\int_{\left(U \cap w U w^{-1}\right) \backslash G} \hat{F}_{\Phi}\left(g, g^{\prime}\right)\left\langle f^{(\lambda)}\left(w^{-1} g\right), \tilde{v}\right\rangle d g \\
& =\int_{\left(w^{-1} U w \cap U\right) \backslash G} \hat{F}_{\Phi}\left(w g, g^{\prime}\right)\left\langle f^{(\lambda)}(g), \tilde{v}\right\rangle d g \\
= & \int_{U \backslash G} \int_{\left(w^{-1} U w \cap U\right) \backslash U} \hat{F}_{\Phi}\left(w u g, g^{\prime}\right)\left\langle f^{(\lambda)}(g), \tilde{v}\right\rangle d u d g .
\end{aligned}
$$

Note that these integrals are absolutely convergent since $\left|\left\langle f^{(\lambda)}(g), \tilde{v}\right\rangle\right| \leqslant\left\|f^{(\lambda)}(g)\right\|\|\tilde{v}\|$, where \|\| is the Hilbert space norm on $\mathcal{V}$. Indeed, $\left\|f^{(\lambda)}\right\|$ is an element of $I(1, \operatorname{Re}(\lambda))$. Thus it remains to show 


\section{LOCAL THETA CORRESPONDENCE AND $R$-GROUPS}

that

$$
\left|a_{\delta}\right|^{-\rho} \int_{\left(U^{\prime} \cap w^{\prime} U^{\prime} w^{\prime-1}\right) \backslash U^{\prime}} F_{\Phi}\left(a_{\delta}, w^{\prime-1} u^{\prime}\right) d u^{\prime}=\int_{\left(w^{-1} U w \cap U\right) \backslash U} \hat{F}_{\Phi}(w u, 1) d u .
$$

We remark that

$$
U=\left\{\left(\begin{array}{cc|cc}
\mathbf{1}_{l} & * & * & * \\
0 & \mathbf{1}_{m} & * & * \\
\hline 0 & 0 & \mathbf{1}_{l} & 0 \\
0 & 0 & * & \mathbf{1}_{m}
\end{array}\right) \in G\right\}
$$

and

$$
w^{-1} U w \cap U=\left\{\left(\begin{array}{cc|cc}
\mathbf{1}_{l} & 0 & 0 & 0 \\
0 & \mathbf{1}_{m} & 0 & * \\
\hline 0 & 0 & \mathbf{1}_{l} & 0 \\
0 & 0 & 0 & \mathbf{1}_{m}
\end{array}\right) \in G\right\},
$$

hence

$$
\left\{u(x, y) u(b) \mid x \in X_{l}, y, b \in M_{l, m}(E)\right\}
$$

is a set of representatives for $\left(w^{-1} U w \cap U\right) \backslash U$. Similarly,

$$
\left\{u^{\prime}\left(x^{\prime}, y^{\prime}\right) u^{\prime}\left(b^{\prime}\right) \mid x^{\prime} \in X_{l}, y^{\prime}, b^{\prime} \in M_{l, m}(E)\right\}
$$

is a set of representatives for $\left(U^{\prime} \cap w^{\prime} U^{\prime} w^{\prime-1}\right) \backslash U^{\prime}$.

First we compute the left-hand side of (6.1), i.e.,

$$
\left|a_{\delta}\right|^{-\rho} \int_{b^{\prime} \in M_{l, m}(E)} \int_{x^{\prime} \in X_{l}} \int_{y^{\prime} \in M_{l, m}(E)} F_{\Phi}\left(a_{\delta}, w^{\prime-1} u^{\prime}\left(x^{\prime}, y^{\prime}\right) u^{\prime}\left(b^{\prime}\right)\right) d y^{\prime} d x^{\prime} d b^{\prime} .
$$

We see that $F_{\Phi}\left(1, w^{\prime-1}\right)$ is equal to

$$
\begin{aligned}
\int_{z \in Z_{\mathbf{m}}} \int_{v_{1}, v_{2}} \omega\left(1, w^{\prime-1}\right) \Phi\left(\begin{array}{cc}
v_{1} & v_{2} \\
0 & z \\
0 & 0 \\
0 & 0
\end{array}\right) \psi\left(\operatorname{tr}\left(v_{1}\right)+\operatorname{tr}(z)\right) d v_{1} d v_{2} d z \\
=|\delta|^{l n} \int_{Z_{\mathbf{m}}} \int_{v_{1}, v_{2}} \int_{v_{3}, v_{4}, v_{5}, v_{6}} \Phi\left(\begin{array}{cc}
v_{3} & v_{2} \\
v_{4} & z \\
v_{5} & 0 \\
v_{6} & 0
\end{array}\right) \\
\quad \times \psi\left(-\operatorname{tr}\left(\delta^{t} \bar{v}_{5} v_{1}\right)\right) \psi\left(\operatorname{tr}\left(v_{1}\right)+\operatorname{tr}(z)\right) d v_{3} d v_{4} d v_{5} d v_{6} d v_{1} d v_{2} d z \\
=|\delta|^{l n-l^{2}} \int_{Z_{\mathbf{m}}} \int_{v_{2}, v_{3}, v_{4}, v_{6}} \Phi\left(\begin{array}{cc}
v_{3} & v_{2} \\
v_{4} & z \\
-\delta^{-1} \mathbf{1}_{l} & 0 \\
v_{6} & 0
\end{array}\right) \psi(\operatorname{tr}(z)) d v_{2} d v_{3} d v_{4} d v_{6} d z .
\end{aligned}
$$

Here $v_{1}, v_{3}, v_{5} \in M_{l}(E), v_{2} \in M_{l, m}(E)$, and $v_{4}, v_{6} \in M_{m, l}(E)$. Hence

$$
\begin{aligned}
\int_{x^{\prime} \in X_{l}} & \int_{y^{\prime} \in M_{l, m}(E)} F_{\Phi}\left(1, w^{\prime-1} u^{\prime}\left(x^{\prime}, y^{\prime}\right)\right) d y^{\prime} d x^{\prime} \\
= & |\delta|^{l n-l^{2}} \int_{X_{l}} \int_{M_{l, m}(E)} \int_{Z_{\mathbf{m}}} \int_{v_{2}, v_{3}, v_{4}, v_{6}} \Phi\left(\begin{array}{ccc}
v_{3} & v_{2} \\
v_{4} & z \\
-\delta^{-1} \mathbf{1}_{l} & 0 \\
v_{6} & 0
\end{array}\right) \\
& \times \psi\left(\operatorname{tr}\left({ }^{t} \bar{v}_{3}-\delta^{t} \bar{v}_{4} v_{6}\right) x^{\prime}+\operatorname{tr}\left({ }^{t} \bar{v}_{2}-\delta^{t} \bar{z} v_{6}\right) y^{\prime}\right) \psi(\operatorname{tr}(z)) d v_{2} d v_{3} d v_{4} d v_{6} d z d y^{\prime} d x^{\prime}
\end{aligned}
$$




\section{A. ICHINO}

$$
\begin{gathered}
=|\delta|^{l n-l^{2}} \int_{X_{l}} \int_{M_{l, m}(E)} \int_{Z_{\mathbf{m}}} \int_{v_{2}, v_{3}, v_{4}, v_{6}} \Phi\left(\begin{array}{cc}
v_{3}-\delta^{t} \bar{v}_{6} v_{4} & v_{2}-\delta^{t} \bar{v}_{6} z \\
v_{4} & z \\
-\delta^{-1} \mathbf{1}_{l} & 0 \\
v_{6} & 0
\end{array}\right) \\
\times \psi\left(\operatorname{tr}\left({ }^{t} \bar{v}_{3} x^{\prime}\right)+\operatorname{tr}\left({ }^{t} \bar{v}_{2} y^{\prime}\right)\right) \psi(\operatorname{tr}(z)) d v_{2} d v_{3} d v_{4} d v_{6} d z d y^{\prime} d x^{\prime} .
\end{gathered}
$$

By Lemma 6.1 and the Fourier inversion formula, this integral is equal to

$$
\begin{aligned}
& |\delta|^{l n-l^{2} / 2} \int_{x \in X_{l}} \int_{Z_{\mathbf{m}}} \int_{v_{4}, v_{6}} \Phi\left(\begin{array}{cc}
\delta x-\delta^{t} \bar{v}_{6} v_{4} & -\delta^{t} \bar{v}_{6} z \\
v_{4} & z \\
-\delta^{-1} \mathbf{1}_{l} & 0 \\
v_{6} & 0
\end{array}\right) \psi(\operatorname{tr}(z)) d v_{4} d v_{6} d z d x \\
& \quad=\left|a_{\delta}\right|^{\rho} \int_{X_{l}} \int_{Z_{\mathbf{m}}} \int_{v_{4}, v_{6}} \omega\left(a_{\delta}^{-1}, 1\right) \Phi\left(\begin{array}{ccc}
x-{ }^{t} \bar{v}_{6} v_{4} & -{ }^{t} \bar{v}_{6} z \\
v_{4} & z \\
\mathbf{1}_{l} & 0 \\
v_{6} & 0
\end{array}\right) \psi(\operatorname{tr}(z)) d v_{4} d v_{6} d z d x
\end{aligned}
$$

Hence the left-side hand of (6.1) is equal to

$$
\begin{array}{r}
\int_{b^{\prime} \in M_{l, m}(E)} \int_{x \in X_{l}} \int_{z \in Z_{\mathbf{m}}} \int_{v_{4} \in M_{m, l}(E)} \int_{v_{6} \in M_{m, l}(E)} \\
\times \Phi\left(\begin{array}{cc}
x-{ }^{t} \bar{v}_{6} v_{4} & x b^{\prime}-{ }^{t} \bar{v}_{6} v_{4} b^{\prime}-{ }^{t} \bar{v}_{6} z \\
v_{4} & v_{4} b^{\prime}+z \\
\mathbf{1}_{l} & b^{\prime} \\
v_{6} & v_{6} b^{\prime}
\end{array}\right) \psi(\operatorname{tr}(z)) d v_{6} d v_{4} d z d x d b^{\prime} .
\end{array}
$$

On the other hand, the right-hand side of (6.1) is equal to

$$
\begin{aligned}
\int_{b \in M_{l, m}(E)} \int_{x \in X_{l}} \int_{y \in M_{l, m}(E)} \int_{z \in Z_{\mathbf{m}}} \int_{v \in M_{l, m}(E)} & \\
& \times \omega(w u(-x,-y) u(b), 1) \Phi\left(\begin{array}{cc}
\mathbf{1}_{l} & v \\
0 & z \\
0 & 0 \\
0 & 0
\end{array}\right) \psi(\operatorname{tr}(z)) d v d z d y d x d b .
\end{aligned}
$$

Calculating directly, we see that

$$
u(-b) u(x, y) w^{-1}\left(\begin{array}{cc}
\mathbf{1}_{l} & v \\
0 & z \\
0 & 0 \\
0 & 0
\end{array}\right)=\left(\begin{array}{cc}
x-b^{t} \bar{y} & x v-b z-b^{t} \bar{y} v \\
{ }^{t} \bar{y} & z+{ }^{t} \bar{y} v \\
\mathbf{1}_{l} & v \\
{ }^{t} \bar{b} & { }^{t} \bar{b} v
\end{array}\right) .
$$

Therefore Equation (6.1) holds.

Corollary 6.3. Let $\kappa \in \hat{R}$. Then

for all $\Phi \in \mathcal{S}$ and $f \in \pi_{\kappa}$.

$$
T(\Phi, f) \in \pi_{\theta(\kappa)}^{\prime}
$$

Proof. Let $i \in \mathfrak{I}$. Then

$$
M\left(w_{i}^{\prime}, \sigma, \lambda\right) T\left(\Phi, f^{(\lambda)}\right)=|\delta|^{n_{i} \lambda_{i}} \omega_{\sigma_{i}}(\delta)^{-1} \epsilon\left(-\lambda_{i}+1 / 2, \sigma_{i}, \psi\right) T\left(\Phi, M\left(w_{i}, \sigma, \lambda\right) f^{(\lambda)}\right)
$$

by Proposition 6.2. Indeed, applying Lemma 5.3 with $d=i-1$, one reduces the case $i>1$ to the case $i=1$. Hence

$$
\mathcal{N}\left(r_{i}^{\prime}, \sigma\right) T(\Phi, f)=\omega_{\sigma_{i}}(\delta)^{-1} \epsilon\left(1 / 2, \sigma_{i}, \psi\right) T\left(\Phi, \mathcal{N}\left(r_{i}, \sigma\right) f\right)
$$

for all $\Phi \in \mathcal{S}$ and $f \in I(\sigma)$. This concludes the proof. 


\section{LOCAL THETA CORRESPONDENCE AND $R$-GROUPS}

Let $\kappa \in \hat{R}$. Combining Lemma 5.4 and Corollary 6.3, we obtain a non-zero $\left(G \times G^{\prime}\right)$-equivariant map

$$
\left.T\right|_{\omega \otimes \pi_{\kappa}}: \omega \otimes \pi_{\kappa} \longrightarrow \pi_{\theta(\kappa)}^{\prime} .
$$

Therefore

$$
\operatorname{Hom}_{G \times G^{\prime}}\left(\omega, \tilde{\pi}_{\kappa} \otimes \pi_{\theta(\kappa)}^{\prime}\right) \simeq \operatorname{Hom}_{G \times G^{\prime}}\left(\omega \otimes \pi_{\kappa}, \pi_{\theta(\kappa)}^{\prime}\right) \neq 0,
$$

and this concludes the proof of Theorem 4.1.

\section{Functoriality}

In this section, we interpret our main result in terms of the Arthur conjecture [Art89b]. Let $W_{F}$ be the Weil group of $F$ and ${ }^{L} G=\hat{G} \rtimes W_{F}$ the $L$-group of $G$. For a discrete series representation $\sigma$ of $L$, let $\varphi_{L}: \mathcal{L}_{F} \rightarrow{ }^{L} L$ denote the Langlands parameter associated to $\sigma$. Here $\mathcal{L}_{F}=W_{F} \times S U_{2}(\mathbb{R})$. By the composition of $\varphi_{L}$ and the embedding ${ }^{L} L \subset{ }^{L} G$, we obtain a Langlands parameter $\varphi$ for $G$. Let $S_{\varphi}$ be the centralizer in $\hat{G}$ of the image $\varphi\left(\mathcal{L}_{F}\right)$, and put $\mathbb{S}_{\varphi}=S_{\varphi} / S_{\varphi}^{0} Z(\hat{G})^{\Gamma}$ with $\Gamma=\operatorname{Gal}(\bar{F} / F)$. Let $\mathbb{S}_{\varphi}^{1}$ be the subgroup of cosets in $\mathbb{S}_{\varphi}$ which act on $S_{\varphi}^{0}$ by inner automorphisms. Then $\mathbb{S}_{\varphi}^{1} \simeq \mathbb{S}_{\varphi_{L}}=\{1\}$ since $L \simeq G L_{n_{1}}(E) \times \cdots \times G L_{n_{t}}(E)$. Therefore, assuming a conjecture in [Art89b, $\S 7$ ], we should have

$$
\mathbb{S}_{\varphi} \simeq R \text {. }
$$

We remark that (7.1) is also consistent with the calculation of $\mathbb{S}_{\varphi}$ in Proposition 2.1 of [Pra00]. If $P$ is a Borel subgroup of $G$, then $(7.1)$ is proved in [Key87, § 2]. Let

$$
\Pi_{\varphi}=\left\{\pi_{\kappa} \mid \kappa \in \hat{R}\right\} \simeq \hat{\mathbb{S}}_{\varphi}
$$

Then $\Pi_{\varphi}$ should be the $L$-packet of $\varphi$. Here the trivial character of $\mathbb{S}_{\varphi}$ corresponds to the $\chi$-generic representation $\pi_{1}$. Similarly, we regard $\varphi^{\prime}=\varphi$ as the Langlands parameter for $G^{\prime}$ and let

$$
\Pi_{\varphi^{\prime}}=\left\{\pi_{\kappa^{\prime}}^{\prime} \mid \kappa^{\prime} \in \hat{R}^{\prime}\right\} \simeq \hat{\mathbb{S}}_{\varphi^{\prime}}
$$

Then the map

$$
\begin{aligned}
\hat{\mathbb{S}}_{\varphi} \longrightarrow \hat{\mathbb{S}}_{\varphi^{\prime}} \\
\kappa \longmapsto \theta(\kappa)
\end{aligned}
$$

defined in $\S 4$ determines the local theta correspondence

$$
\begin{aligned}
\Pi_{\varphi} & \longrightarrow \Pi_{\varphi^{\prime}} \\
\pi & \longmapsto \theta(\pi) .
\end{aligned}
$$

Remark 7.1. This interpretation of Theorem 4.1 is consistent with a conjecture of Prasad [Pra00]. Note that he uses the extended $L$-packet by Vogan [Vog93], but it suffices to consider the usual $L$-packet in this case. Indeed, $\varphi$ is not a Langlands parameter for the non-quasi-split inner form of $G$.

\section{ACKnowledgements}

The author expresses gratitude to Professor Tamotsu Ikeda for his advice and encouragement. The author would like to thank Dr. Kaoru Hiraga for pointing out an ambiguity in an earlier version of the main theorem, and Professor Dipendra Prasad for comments on his conjecture. Thanks are also due to the referee for helpful comments. 


\section{LOCAL THETA CORRESPONDENCE AND $R$-GROUPS}

\section{REFERENCES}

Art89a J. Arthur, Intertwining operators and residues. I. Weighted characters, J. Funct. Anal. 84 (1989), $19-84$.

Art89b J. Arthur, Unipotent automorphic representations: conjectures, Astérisque 171-172 (1989), 13-71.

GJ72 R. Godement and H. Jacquet, Zeta functions of simple algebras, Lecture Notes in Mathematics, vol. 260 (Springer-Verlag, Berlin, 1972).

Gol94 D. Goldberg, Some results on reducibility for unitary groups and local Asai L-functions, J. Reine Angew. Math. 448 (1994), 65-95.

Gol95 D. Goldberg, R-groups and elliptic representations for unitary groups, Proc. Amer. Math. Soc. 123 (1995), 1267-1276.

HKS96 M. Harris, S. S. Kudla and W. J. Sweet, Jr., Theta dichotomy for unitary groups, J. Amer. Math. Soc. 9 (1996), 941-1004.

How79 R. Howe, $\theta$-series and invariant theory, in Automorphic forms, representations and L-functions, Proc. Symp. Pure Math., vol. 33-1 (Amer. Math. Soc., Providence, RI, 1979), 275-285.

Key87 C. D. Keys, L-indistinguishability and R-groups for quasi-split groups: unitary groups in even dimension, Ann. Sci. École Norm. Sup. 20 (1987), 31-64.

KS80 A. W. Knapp and E. M. Stein, Intertwining operators for semisimple groups, II, Invent. Math. 60 (1980), 9-84.

Kud86 S. S. Kudla, On the local theta-correspondence, Invent. Math. 83 (1986), 229-255.

Kud94 S. S. Kudla, Splitting metaplectic covers of dual reductive pairs, Israel J. Math. 87 (1994), 361-401.

MVW87 C. Mœglin, M.-F. Vignéras and J.-L. Waldspurger, Correspondances de Howe sur un corps p-adique, Lecture Notes in Mathematics, vol. 1291 (Springer-Verlag, Berlin, 1987).

Pra00 D. Prasad, Theta correspondence for unitary groups, Pacific J. Math. 194 (2000), 427-438.

Sha90 F. Shahidi, A proof of Langlands' conjecture on Plancherel measures; complementary series for p-adic groups, Ann. Math. 132 (1990), 273-330.

Sil78 A. J. Silberger, The Knapp-Stein dimension theorem for p-adic groups, Proc. Amer. Math. Soc. 68 (1978), 243-246

Sil79a A. J. Silberger, Correction: The Knapp-Stein dimension theorem for p-adic groups, Proc. Amer. Math. Soc. 76 (1979), 169-170.

Sil79b A. J. Silberger, Introduction to harmonic analysis on reductive p-adic groups (Princeton University Press, Princeton, NJ, 1979).

Vog93 D. A. Vogan, Jr., The local Langlands conjecture, in Representation theory of groups and algebras (Amer. Math. Soc., Providence, RI, 1993), 305-379.

Wal90 J.-L. Waldspurger, Démonstration d'une conjecture de dualité de Howe dans le cas p-adique, $p \neq 2$, Festschrift in honor of I. I. Piatetski-Shapiro on the occasion of his sixtieth birthday, Part I (Weizmann, Israel, 1990), 267-324.

Atsushi Ichino ichino@sci.osaka-cu.ac.jp

Department of Mathematics, Graduate School of Science, Osaka City University, 3-3-138 Sugimoto, Sumiyoshi-ku, Osaka 558-8585, Japan 